\title{
LAS PARADOJAS DE LA RESISTENCIA DURANTE EL PROCESO DE CURA SEGÚN LA PERSPECTIVA PSICOANALÍTICA
}

\author{
Leydi Damaris Restrepo Giraldo ${ }^{1}$ \\ Corporación Universitaria Minuto de Dios, Colombia \\ leydamaris@gmail.com \\ ORCID: 0000-0003-0154-5099
}

DOI: 10.17533/udea.affs.v16n31a06

\section{Resumen}

El presente artículo aborda las paradojas de la resistencia en la dirección de la cura psicoanalítica, vista en términos de una doble funcionalidad aparentemente excluyente puesto que permite la cura, pero también atenta contra ella, produciendo así una tensión entre enfermedad (repetir) y salud (recordar). Para ello se sirve de

diversos textos de Freud y Lacan que permiten caracterizar sus respectivas posturas sobre el tema, a fin de generar una reflexión sobre ellas para tratar de comprender qué permite explicar la ambivalencia en este concepto.

Palabras clave: Cura, Resistencia, Paradoja, Repetir, Recordar.

\section{THE PARADOXES OF RESISTANCE DURING THE PROCESS OF TREATMENT ACCORDING TO THE PSYCHOANALYTIC PERSPECTIVE}

\section{Abstract}

This paper addresses the paradoxes terms of a double functionality apof resistance in the direction of the parently mutually exclusive because psychoanalytic treatment taken in it allows treatment, but it also attacks

1 Profesional en Psicología, con experiencia en programas de atención psicosocial y consulta psicológica privada. 
it, so that it produces a tension between illness (repeating) and health (remembering). To do so, it takes into account some Freudian and Lacanian texts that allow to characterize their stances on this subject in order to generate a reflexion on them to try to understand the explanation of such ambivalence.

Keywords: treatment, resistance, paradox, repeating, remembering.

\section{LES PARADOXES DE LA RÉSISTANCE PENDANT LE PROCESSUS DE CURE SELON LA PERSPECTIVE PSYCHANALYTIQUE}

\section{Résumé}

Cet article aborde les paradoxes de la résistance dans l'orientation de la cure psychanalytique, considérant une double fonction apparemment exclusive : elle rend possible la cure mais en même temps elle l'attaque, produisant ainsi une tension entre la maladie (répéter) et la santé (remémorer). Pour ce faire, l'on examine divers textes de Freud et de Lacan permettant de caractériser leurs positions à ce sujet. Le but est de susciter une réflexion sur ces jugements afin d'essayer de comprendre la source de l'ambivalence dans ce concept.

Mots-clés : cure, résistance, paradoxe, répéter, remémorer.

Recibido: 02/07/2018 • Aprobado:19/01/2019 


\section{Introducción}

El propósito del presente artículo reflexivo es desarrollar una indagación sobre las paradojas de la resistencia durante el proceso de cura según la perspectiva psicoanalítica. Para ello se realiza una caracterización de la postura del psicoanálisis sobre el tema y se genera una reflexión sobre ella a fin de tratar de comprender qué permite explicar la paradoja de este concepto, con el objetivo de proponer construcciones teóricas que sirvan a estudiantes, psicólogos o psicoanalistas interesados en el asunto.

Se ofrece, así mismo, una definición de los conceptos cura analítica y sus desarrollos en psicoanálisis, como también del concepto de resistencia; se realiza el planteamiento de la paradoja en la resistencia, una contextualización sobre el descubrimiento de la importancia de la misma, las formas que adopta y sus niveles; a su vez, se delimitan algunos terrenos en los cuales ésta se presenta durante el proceso de análisis, se exponen algunas explicaciones que permiten comprender dicha paradoja, se plantean algunas preguntas sobre la resistencia hoy y fuera del discurso psicoanalítico, para finalmente exponer algunas conclusiones.

\section{Resistencia y cura}

Para dar comienzo a la presente reflexión sobre las paradojas de la resistencia, hay que decir inicialmente que dentro de la teoría psicoanalítica el fenómeno de la resistencia está íntimamente relacionado con la cura en la medida en que se supone que el analizante, a lo largo de su trabajo analítico, usualmente se opone, de manera inconsciente, a ponerle fin a su padecimiento subjetivo y procura mantenerse en él. En ese sentido, una cura analítica sería el producto de la conquista que se ha logrado sobre las resistencias, pues se les ha vencido durante el proceso de análisis y han caído los obstáculos que impedían saber de sí y saber del inconsciente. A propósito de esto, Freud lo indica de la manera siguiente: 
La cura analítica impone a médico y enfermo un difícil trabajo que es preciso realizar para cancelar unas resistencias internas. Mediante la superación de estas, la vida anímica del enfermo se modifica duraderamente, se eleva a un estadio más alto del desarrollo y permanece protegida frente a nuevas posibilidades de enfermar. (Freud, 2007a/1916-1917, p. 410).

Nótese que, para acceder durante el análisis a los contenidos inconscientes, se precisa de la superación de las resistencias a fin de conducir al sujeto hacia una posición en la cual haya dejado de resistirse a saber sobre sí y sobre su realidad íntima, para que logre acceder por sí mismo a ese saber reprimido: lo inconsciente. La cura, entonces, implica, entre otros fenómenos clínicos, la caída de las resistencias a las cuales el paciente recurría para aferrarse a la repetición de sus síntomas y al sufrimiento que le causaban. De esta manera se espera que la cura, por medio del vencimiento de dichas resistencias, produzca un levantamiento y cese de los síntomas como formas sustitutivas de satisfacción pulsional, que son vivenciados como sufrimiento, para dar paso a un grado mayor de bienestar posible en la vida del analizante.

De lo anteriormente dicho se extrae que el proceso de cura implica cuestiones diversas con respecto a la resistencia, que serán expuestas a continuación de manera más expresa mediante el planteamiento de la misma y su carácter paradojal.

\section{La resistencia y el planteamiento de su paradoja}

El término resistencia es de uso común entre psicólogos y analistas, además es un concepto importante en la teoría psicoanalítica y un fenómeno clínico que se ha presentado de manera sistemática durante el proceso psicoanalítico en la dirección de la cura, tanto en los pacientes de antaño como en los de hoy, aunque no siempre es percibido a tiempo ni orientado adecuadamente. Por esa razón, no debe perderse de vista que "durante una cura, la resistencia aparece repetidas veces (...)" (Velásquez, 2006, p. 32) y tanto analista como paciente deben contar con ella durante todo el proceso analítico.

Ya decía Freud en "Resistencia y represión" que "cuando emprendemos el restablecimiento de un enfermo para liberarlo de sus 
síntomas patológicos, él nos opone una fuerte, una tenaz resistencia, que se mantiene durante todo el tratamiento" (2007a/1916-1917, p. 262). Este planteamiento se articula con un fenómeno que es observable en la práctica clínica, pues los pacientes y los analistas saben que es necesaria la superación continuada de innumerables obstáculos que se oponen permanentemente al intento de cura y al levantamiento de los síntomas. La cura en psicoanálisis consiste en producir un saber que tenga el carácter de verdad constitutiva sobre la cual conscientemente no se tiene noticia. Esta situación actualiza permanentemente el fenómeno de la resistencia en el contexto clínico y le otorga vigencia, más de un siglo después de ser descubierta por Freud en el dispositivo analítico y descrita en los estudios que realizó junto con Breuer sobre la histeria y que expone en su libro "Estudios sobre la Histeria" (2007a/1893-1895).

De acuerdo con lo anterior, la resistencia puede ser un elemento que, en un primer momento, hace afrenta a la dirección de la cura y puede ser un factor determinante en la deserción de un paciente de su proceso de análisis, o un motivo por el cual los resultados alcanzados no sean relevantes ni significativos con respecto a las expectativas, pues las censuras sobre lo reprimido impiden que ello acceda a la conciencia de manera nítida; así, algo resiste y aparece como dique u obstáculo que se opone a la analizabilidad. Es el mismo Freud el que considera que la causa de la resistencia está en el núcleo de lo reprimido, que en la cura es lo que hace límite a la analizabilidad (Velásquez, 2006, p. xix) , lo cual quiere decir que la resistencia se opone al ejercicio necesario para la cura; se requiere entonces tomar noticia de ella, percatarse de sus múltiples formas de aparición y traducir los contenidos susceptibles de ello que vayan surgiendo durante el proceso de análisis en lucha con los procesos psíquicos de la represión que se le oponen y que se explican como última causa que hace oposición.

\section{Concepto de resistencia}

Según Freud, citado en Lacan, la resistencia, de un lado, es "Todo lo que destruye/suspende/altera/la continuación del trabajo [analítico] (...)" (Lacan, 1988b/1953-1954, p. 59). Definición, que en otras palabras significa: cualquier obstáculo, dificultad o impedimento que 
haga presencia a lo largo de la labor analítica entre analista y paciente, logrando perturbarla, detenerla o pararla, merece el calificativo de resistencia. Sin embargo, es importante anotar que ésta se presenta muchas veces de manera inconsciente para el paciente y no siempre resulta fácil su vencimiento.

Pero nótese también que este mismo autor nos ofrece otra mirada sobre las resistencias que parece contradecir su definición de las mismas, diciendo que éstas "contienen tanto del material más importante del pasado del enfermo y lo espejan de manera tan convincente que se convierten en los mejores soportes del análisis si una técnica diestra saber darles el giro correcto" (Freud, 2007a/1916-1917, p. 266). La resistencia, entonces, se revela no solo como un obstáculo, como generalmente es concebida, sino también como posibilidad y oportunidad de camino hacia la dirección de la cura, por cuanto proporciona un saber de aquello latente, oscuro y desconocido para el paciente al aportar elementos diversos y cruciales sobres su historia pasada. En este sentido conviene preguntarse: ¿qué permite explicar la paradoja del concepto resistencia en la dirección de la cura dentro de la teoría psicoanalítica? Dicha pregunta es pertinente ya que, como se ha visto, implica simultáneamente dos opuestos: es obstáculo y palanca, lo cual se presenta como una aparente contradicción, una ambivalencia que reúne cosas opuestas y aparentemente excluyentes. De un lado, la resistencia como obstáculo pretende repetir lo sintomático, permanecer en ello e impedir la continuación del análisis; y de otro, la resistencia como palanca puede permitir, una vez superada, la recordación de lo reprimido, hacerlo consciente y posibilitar la cura.

\section{Descubrimiento de la importancia de la resistencia, las formas que adopta y sus niveles}

Freud descubrió la importancia de la resistencia como fenómeno clínico durante su trabajo analítico con pacientes histéricas e hizo alusión a ello en los "Estudios sobre la histeria", más específicamente en los siguientes dos textos: “Señorita Elisabeth von R" (2007b/1893-1895) y 
"Sobre la psicoterapia de la histeria" (2007a/1893-1895). En el primer caso, Elizabeth era una paciente de Freud que tenía 24 años y padecía dolores en las piernas debidos a una histeria; durante su trabajo psicoterapéutico con ella, para producir una recuperación, él se vio enfrentado a superar ciertas dificultades en el proceso de evocación de los recuerdos reprimidos que estaban asociados a los síntomas que presentaba la paciente. De allí dedujo que traer esas experiencias reprimidas a la conciencia parecía ser una tarea que presentaba grandes dificultades en ella, dificultades que Freud de otra manera llamó resistencias. Con respecto a este caso con la señorita von $\mathrm{R}$, él dice lo siguiente:

En el curso de este difícil trabajo empecé a atribuir una significación más profunda a las resistencias que la enferma mostraba a reproducir sus recuerdos, y a compilar con cuidado las ocasiones a raíz de las cuales aquella se denunciaba de un modo particularmente llamativo. (2007b/1893-1895, p. 168).

En el segundo caso, Freud también hace alusión al descubrimiento de la importancia de la resistencia, en un texto llamado "Sobre la psicoterapia de la histeria", en el que la menciona como fenómeno para aludir a las dificultades que los pacientes presentaban cuando se esforzaban por recordar las situaciones que estaban asociadas o en las cuales se habían producido los síntomas patógenos. Sin embargo, con esfuerzo y empeño del paciente, ayudado del terapeuta, cuenta que se lograba finalmente que afloraran algunos recuerdos que ofrecían claridad con respecto a las causas primeras de la formación patógena del síntoma (Freud, 2007b/1893-1895, p. 275). Es así como Freud se da cuenta de que para lograr esas reconstrucciones y acceder a lo reprimido "tenía que superar en el paciente una fuerza que contrariaba el devenir consciente (recordar) de las representaciones patógenas" (p. 275). Esa fuerza que contrariaba el devenir consciente podría ser pensada como la forma en que Freud nombra la resistencia, en este caso, como un obstáculo para recordar lo reprimido, que él denomina como representaciones patógenas.

Ha de tenerse en cuenta que la resistencia se puede presentar en diversas formas, las cuales se presentan a continuación. 
Según Freud, la resistencia no presenta una única forma y ésta cambia constantemente su aparición durante el proceso psicoterapéutico. La siguiente es una amplia descripción que él ofrece para dar una idea general de los múltiples matices que puede adoptar durante el curso de un análisis.

Agreguen a esto que el enfermo explota, convirtiéndolas en un obstáculo, todas las contingencias que surgen durante el tratamiento, todo suceso externo que pueda distraer de la tarea, toda manifestación pronunciada en su círculo por una autoridad hostil al análisis, una enfermedad orgánica casual o que complique la neurosis, y que él mismo aprovecha como motivo para ceder en su empeño cualquier mejoría de su estado, y tendrán un cuadro aproximado, aunque todavía incompleto, de las formas y medios a que recurre la resistencia, en lucha contra la cual trascurre todo análisis. (2007a/1916-1917, pp. 266-267).

Si bien esta es una descripción bastante amplia de los diversos matices que adopta la resistencia y de los que se puede valer para cumplir el cometido de estorbar el direccionamiento de la cura, ésta comporta, pues, un carácter pluriforme; se da ya sea en forma de eventualidades, críticas, obstáculos, distracciones o aparentes accidentes imprevistos. Se presenta de manera variable, móvil y dinámica, y puede dirigirse al médico, al dispositivo o a la teoría. Por ejemplo, una aparente y pronta mejoría puede ser un medio útil para la resistencia. Sin embargo, más allá de la diversidad en la que se presenta la resistencia, en tanto fundamental o de base, es una constante que permanece durante el proceso psicoterapéutico y ese carácter constante subsiste a cualquier obstáculo o impedimento, en tanto fuerza de oposición al tratamiento analítico y a la cura de los síntomas.

Pero no solamente la resistencia es constante durante todo el análisis, sino que, además, también puede adoptar diversas magnitudes en su fuerza, cambiando frecuentemente el empuje de la misma, según la distancia en la que se halle la conciencia del paciente de lo inconsciente reprimido. Con respecto a estos niveles de la resistencia Freud dice lo siguiente:

En efecto, en el curso de un tratamiento la intensidad de la resistencia varía de continuo; aumenta cada vez que nos aproximamos a un 
tema nuevo, llega a su máxima fuerza en el ápice de la elaboración de este y vuelve a desbaratarse cuando se lo finiquita. (2007a/19161917, p. 268).

Con lo anteriormente dicho se muestra también que el empuje de la resistencia posee una magnitud directamente proporcional a lo que Freud llama la aproximación a un nuevo tema, lo cual no es más que el acercamiento a los contenidos reprimidos; dicho de otro modo, la resistencia es de mayor intensidad cuando el paciente se aproxima a materiales inconscientes que le resultan dolorosos en grado sumo y que le son desconocidos a su conciencia, puesto que son la parte más delicada, a razón de estar asociados a las representaciones que causan la formación de los síntomas. Una vez se hagan conscientes para él estos materiales dolorosos, la resistencia disminuye o desaparece completamente, posibilitando, a partir de saber sobre la naturaleza de la misma, la continuidad del tratamiento.

\section{Tres terrenos de la resistencia y la explicación de su paradoja}

En la "19a Conferencia. Resistencia y represión", Freud plantea tres escenarios en los que hace su aparición el fenómeno de la resistencia a lo largo de la labor analítica: el primero de estos terrenos es el de la asociación libre, el segundo alude al mecanismo de racionalización, y el tercero es el de la transferencia.

Con respecto al surgimiento de la resistencia durante el proceso de la asociación libre, Freud dice lo siguiente: "Cuando fijamos esta regla fundamental, lo primero que conseguimos es que se convierta en el blanco de ataque de la resistencia. El enfermo procura evadirse por todos los medios de sus imperativos" (2007a/1916-1917, p. 263). En ese sentido, parece ser que cuando el paciente tiene dificultad para decir espontáneamente y sin críticas lo que vaya emergiendo a su conciencia durante la sesión de análisis, está resistiéndose a asociar libremente para dar lugar al surgimiento del inconsciente.

Por tanto, para que se produzca la asociación libre durante la sesión de análisis se le pide al analizante lo siguiente: 
Que se ponga en un estado de calma observación de sí sin reflexión, y nos comunique todas las percepciones interiores que pueda tener en ese estado -sentimientos, pensamientos, recuerdos-, en la secuencia en que emergen dentro de él. Le advertimos de una manera expresa que debe resignar cualquier motivo que le haría practicar una selección o exclusión entre las ocurrencias: que eso es demasiado desagradable o indiscreto para decirlo, o que es demasiado trivial, no viene al caso, o es disparatado y no hace falta decirlo (...) y le aseguramos que el resultado del tratamiento, sobre todo su duración, depende de la escrupulosidad con que obedezca a esta regla técnica fundamental del análisis. (2007a/1916-1917, p. 263).

Sin embargo, en muchos casos los pacientes evitan hablar de ciertos temas en el espacio terapéutico, ya sea por pudor, vergüenza, crítica, temor $\mathrm{u}$ otras razones a veces desconocidas para ellos mismos. Para superar estas formas de la resistencia en el terreno de la asociación libre, el analista deberá hacerlas evidentes frente al paciente y redirigirlas adecuadamente para ponerlas al servicio del tratamiento y proseguir la búsqueda de la cura analítica.

Pero, una vez vencida la resistencia en el primer terreno, es decir, en la asociación libre, ésta se traslada al segundo, al cual Freud dio por nombre resistencia intelectual y explica de la siguiente manera:

Baste con saber que al final se logra, a fuerza de decisión y de tenacidad, arrancarle a la resistencia una cierta cuota de obediencia a la regla técnica fundamental, y entonces ella se vuelca a otro ámbito. Aparece como resistencia intelectual, lucha con argumentos, se hace fuerte en las dificultades e inverosimilitudes que el pensamiento normal, pero no instruido, halla en las doctrinas analíticas. (2007a/1916-1917, p. 264).

La resistencia intelectual o racionalización se presenta en el paciente bajo la forma de críticas contra el psicoanálisis o el analista, con matices de desconfianza frente a la efectividad del tratamiento; también en forma de duda con respecto al éxito que pueda alcanzar el paciente durante el mismo. La resistencia en la racionalización del lado del paciente también presenta esta forma: "Si algo no le viene bien, puede defenderse contra eso con mucha agudeza y aparecer muy crítico; si algo le conviene, puede mostrarse muy crédulo" (2007a/1916- 
1917, p. 268) y aparentar interés, pero luego la persistencia de los síntomas hará notar que no ha habido una real mejoría.

Este terreno particular de la resistencia ofrece, sin duda, una singular dificultad y se requiere cierta pericia del analista para sortearla; sin embargo, para Freud hay un terreno en el cual la resistencia se presenta con mayor complejidad y dificultad. A propósito de esto afirma lo siguiente.

Las resistencias intelectuales no son las peores; siempre se sale vencedor de ellas. Pero el paciente se las compone también, mientras permanece dentro del marco del análisis, para producir resistencias cuyo vencimiento se cuenta entre las más difíciles tareas técnicas. En lugar de recordar repite unas actitudes y mociones afectivas de su vida que, por medio de la llamada "transferencia", pueden emplearse para resistirse al médico y a la cura. (2007a/1916-1917, p. 265).

Es así como la resistencia en el paciente se traslada al tercer y último terreno, que Freud llama resistencia de transferencia, en la cual el paciente pone en la persona del analista actitudes afectivas, recuerdos y material de su vida temprana en relación con sus representaciones familiares de orden primordial, es decir, las figuras que han sido representativas o significativas en la vida del paciente. En la resistencia de transferencia, el paciente revive con el analista su pasado vivido, su historia infantil, su vida pretérita y repite con él sentimientos hacia personas significativas de su infancia, hasta ese entonces desconocidos por la conciencia. Es de anotar que este tipo de resistencia, es decir la transferencia, es el mayor obstáculo en la dirección de la cura pues "en el análisis la trasferencia nos sale al paso como la más fuerte resistencia al tratamiento" (Freud, 2005/1912, p. 99).

Al mismo tiempo, es preciso también anotar a este respecto que la resistencia de trasferencia, según Freud, puede aparecer sobre el analista en forma hostil o negativa y en forma erótica o positiva. La primera puede surgir en forma de críticas, afectos hostiles, desconfianza o proyección de sentimientos agresivos. La segunda, bajo formas de idealización o admiración exageradas hacia la persona del analista, bajo un modelo de perfección, o la exageración de afectos tiernos, sexuales o amorosos. Ambas pueden ser confesadas o no al analista, presentarse 
como una actitud, una conducta, o permanecer veladas. El paciente puede experimentarlas, pero no confesarlas ni hacerlas explicitas. En este sentido, "Es preciso decidirse a separar una trasferencia 'positiva' de una 'negativa', la trasferencia de sentimientos tiernos de la de sentimientos hostiles, y tratar por separado ambas variedades de trasferencia sobre el médico" (Freud, 2005/1912, p. 102). La recomendación que da el mismo Freud es recibirlas y darles lugar. Encausarlas bajo el dispositivo analítico de la palabra, la asociación libre, la interpretación y el sentido, para interrogarlas, descubrir su origen, su causa primaria inconsciente y hacerlas conscientes para el paciente; pues la transferencia se sirve de ambos tipos de resistencia y es necesario anoticiarse de eso para contar con ello y volverlas objeto del tratamiento.

Se debe tener en cuenta, según Freud, que:

Para la finalización de una cura analítica, la transferencia misma tiene que ser desmontada; y si entonces sobreviene o se mantiene con éxito, no se basa en la sugestión, sino en la superación de resistencias ejecutada con su ayuda y en la transformación interior promovida en el enfermo. (2007a/1916-1917, p. 412).

Es decir, en el análisis se produce la cura analítica cuando las resistencias han sido desmanteladas una tras otra y se logra ir más allá de ellas para acceder al inconsciente. Una vez derribadas algunas de ellas, pueden reaparecer en otro lugar, surgir de otra manera o cobrar formas nuevas. Su vencimiento requiere tiempo, paciencia, pericia del analista, y no se da mediante la sugestión efectuada sobre paciente, sino con su colaboración, su palabra, los cambios y las mutaciones producidas en su inconsciente.

\section{Paradojas de la resistencia}

Expuestos ya los tres terrenos de la resistencia, ahora se procederá a la tarea de abordar algunas explicaciones que permiten comprender su carácter paradojal, principalmente en el tercer terreno que Freud llamó terreno de transferencia, es decir, la transferencia vista como una forma de resistencia, la más difícil de vencer durante el tratamiento en la dirección de la cura. 
Con respecto a la primera paradoja, Freud señala lo siguiente: "La resistencia acompaña todos los pasos del tratamiento; cada ocurrencia singular, cada acto del paciente, tiene que tomar en cuenta la resistencia, se constituye como entre las fuerzas cuya meta es la salud y aquellas, ya mencionadas, que las contrarían" (2005/1912, p. 101). Esta paradoja de la resistencia se explica, entonces, como un compromiso de lucha entre dos fuerzas opuestas durante el análisis, unas que alimentan la repetición, la fijación al malestar subjetivo, al sufrimiento; y otras fuerzas que pujan por acceder a un grado de bienestar singular de acuerdo a las posibilidades del sujeto que se analiza, mediante un gran esfuerzo por recordar y saber sobre sí. Aquí se presenta una lucha de dos opuestos: salud y enfermedad.

La segunda paradoja en Freud nos plantea que: "A primera vista, parece una gigantesca desventaja metódica del psicoanálisis que en él la trasferencia, de ordinario la más poderosa palanca del éxito, se mude en el medio más potente de resistencia" (2005/1912, p. 99). Es así como en la transferencia pueden darse varios movimientos. Ésta puede ser motor de cura, en tanto el paciente manifiesta aquí lo que no recuerda conscientemente. La transferencia es una fuente de saber y posibilita la interpretación sobre ella si está dirigida adecuadamente, lo cual la torna en palanca que ayuda a mostrar lo que antes estaba oculto, develar lo inconsciente y arrojar luz sobre lo más íntimo; pero también puede transformarse en desventaja para la cura si es demasiado hostil y no se tiene un direccionamiento adecuado de la misma. Esto hace entonces que se convierta en el medio más potente que obstaculiza la continuidad del análisis.

Pero pese a todo lo anterior, es claro que la resistencia de transferencia se convierte en un elemento necesario y crucial dentro del análisis para la dirección de la cura, pues aquí se libran los verdaderos combates entre paciente-analista y se exponen los contenidos anímicos inconscientes más cruciales, a los cuales se les busca una salida deseable para el paciente.

La tercera paradoja en Freud es planteada de la siguiente manera:

(...) superamos la trasferencia cuando demostramos al enfermo que sus sentimientos no provienen de la situación presente y no valen para la persona del médico, sino que repiten lo que a él le 
ocurrió una vez, con anterioridad. De tal manera lo forzamos a mudar su repetición en recuerdo. Y entonces la trasferencia que, tierna u hostil, en cualquier caso parecía significar la más poderosa amenaza para la cura, se convierte en el mejor instrumento de ella, con cuya ayuda pueden desplegarse lo más cerrados abanicos de la vida anímica". (2007b/1916-1917, p. 413).

La transferencia como una forma de la resistencia contiene, al mismo tiempo, dos elementos opuestos entre sí al análisis: amenaza y posibilidad de cura. Una posible salida a este obstáculo, que ofrece el mismo Freud al respecto, es mudar lo que el paciente repite en rememoración consciente para acceder a los contenidos anímicos más recónditos que permanecen ocultos y alejados del yo consciente, es decir, lo reprimido, mudando esa repetición sintomática en recuerdo consciente que genera alivio subjetivo y bienestar.

Es necesario aclarar que, para Lacan, a diferencia de Freud, la resistencia recae sobre el analista cuando éste interviene desde su propia persona. Lo asevera cuando dice que "no hay otra resistencia al análisis sino la del analista mismo" (2002, p. 575), sobre el cual podría decirse que yerra durante el análisis en la dirección de la cura o fija una resistencia cuando produce interpretaciones desatendiendo a la transferencia o en momentos en los cuales se deja orientar por sus prejuicios personales que le impiden situarse con imparcialidad ante ella, a fin de reconocer la verdad que el analizante allí le expresa.

Con respecto a la cuarta paradoja de la resistencia que se puede ubicar en Lacan, podría decirse que está situada en la transferencia y es señalada por este autor como el mayor obstáculo en la dirección de la cura. Lo señala de la siguiente manera:

Lo que Freud nos indica, desde un principio, es que la transferencia es esencialmente resistente, Ubertragungswiderstand. La transferencia es el medio por el cual se interrumpe la comunicación del inconsciente, por el que el inconsciente se vuelve a cerrar. Lejos de ser el momento de la transmisión de poderes al inconsciente, la transferencia es al contrario su cierre. Esto es esencial para marcar la paradoja que se suele expresar con bastante frecuencia mediante la indicación siguiente, que también encontramos en el texto de Freud -el analista debe esperar la transferencia para empezar a dar la interpretación. (2003/1964, p. 136). 
Si bien la transferencia en Lacan presenta una cualidad dual, es decir, ella cierra y abre el inconsciente, dos situaciones aparentemente excluyentes con respecto al direccionamiento de la cura, si se le mira cuidadosamente, el analista debe esperar a que la transferencia esté y produzca aparentemente un cierre o interrupción del análisis para dar inicio a la apertura del inconsciente con su labor de interpretación sobre ella, haciendo uso de los materiales diversos que el analizante allí aflora, rememora y evoca.

Continuando con la explicación de las paradojas de la resistencia durante el proceso analítico, se dirá que este fenómeno ambivalente es inherente a la clínica misma, pues el mismo Freud decía que cuando la resistencia aparecía durante el proceso de análisis, se creía que se estaba muy lejos de la cura analítica, pero era justo en ese momento donde precisamente se encontraba uno más próximo a ella, ya que durante su auge y desarrollo es precisamente cuando se descubren los contenidos reprimidos de los cuales ella se alimenta. A razón de lo anterior, puede decirse que la resistencia, además de ser un elemento paradójico, como quedó expuesto, también es un componente necesario para alcanzar la cura analítica, así lo expresa Freud en el texto Recordar, repetir y reelaborar (2005/1914):

A menudo me han llamado a consejo para casos en que el médico se quejaba de haber expuesto al enfermo su resistencia, a pesar de lo cual nada había cambiado o, peor, la resistencia había cobrado más fuerza y toda la situación se había vuelto aún menos trasparente. La cura parecía no dar un paso adelante. Luego, esta expectativa sombría siempre resultó errónea. Por regla general, la cura se encontraba en su mayor progreso; sólo que el médico había olvidado que nombrar la resistencia no puede producir su cese inmediato. Es preciso dar tiempo al enfermo para enfrascarse en la resistencia, no consabida para él; para reelaborarla \{durcharbciten\}, vencerla prosiguiendo el trabajo en desafío a ella y obedeciendo a la regla analítica fundamental. (pp. 156-157).

De otro lado, siguiendo a Lacan respecto a la dirección de la cura, para articularlo con lo dicho anteriormente por Freud, permite entender que la resistencia, para él aparece durante el proceso de análisis allí donde no puede decirse más, donde se agotan las palabras para nombrar el deseo, para capturarle en las letras, y esto es estructural al 
aparato psíquico durante la práctica clínica; puesto que no todo puede ser dicho durante un proceso de análisis, algo quedará por fuera, algo escapará al esfuerzo por ser dicho. Así lo explicita cuando dice:

Que, puesto que no se pone ningún obstáculo a la confesión del deseo, es hacia eso hacia donde el sujeto es dirigido e incluso canalizado. Que la resistencia a esa confesión, en último análisis, no puede consistir aquí en nada sino en la incompatibilidad del deseo con la palabra. (Lacan, 2002, p. 621).

\section{La cura en psicoanálisis}

El concepto de cura en psicoanálisis es harto complejo, pues implica una conceptualización que no logra ser definitiva. Tanto en Freud como en Lacan, y en otros autores, son diversas las proposiciones que se erigen en torno a ella; en la medida en que la teoría del psicoanálisis va desarrollándose, va modificándose dicha concepción, adquiere diversos sentidos y significaciones. Así mismo, es posible entrever que la cura, en psicoanálisis, por distintas razones se topa con limitaciones, a la vez que se encuentra con posibilidades, según los retos que se impongan a la clínica y teoría psicoanalíticas en su evolución.

Por un lado, Freud plantea en 1904 una cura relativa a los efectos que el psicoanálisis logra en la capacidad del sujeto de disponer de su libido en términos prácticos; ésta, si se quiere una definición pragmática de la cura, entendida como capacidad de rendimiento y capacidad de disfrute. En este punto la capacidad de amor y trabajo se convierten en indicadores de la cura psicoanalítica; a propósito de esto Freud afirma:

No puede postularse para el tratamiento ninguna otra meta que una curación práctica del enfermo, el restablecimiento de su capacidad de rendimiento y de goce. En caso de que la cura o sus resultados sean incompletos, se obtiene básicamente una importante mejoría del estado psíquico general, mientras que los síntomas pueden persistir, aunque su importancia habrá disminuido para el enfermo y no le pondrán el marbete de tal. (2007/1903-1904, p. 241). 
Con esta definición Freud nos advierte que esa versión de la cura es incompleta, dejando restos sintomáticos y modificando la relación con el síntoma en lugar de su levantamiento total; es decir, que en lugar del esperado e ideal total levantamiento de los síntomas que Freud ha planteado ya a partir de hacer consciente lo inconsciente, aparece ahora la reducción sintomática, que permite una mejoría, más no una cura radical.

De otro lado, Freud plantea en 1932-1933 en la "31ª Conferencia. La descomposición de la personalidad psíquica" la cura en la rúbrica del yo y el ello, esta concepción se agrega planteando la relación que se establece entre la cura y las tres instancias psíquicas de su segunda tópica freudiana: ello, yo y superyó. Desde esta perspectiva la cura se produce a partir de un fortalecimiento del yo con respecto al superyó, produciéndose así una distancia que le permite al yo apropiarse, según Freud, de "nuevos fragmentos del ello" (2007/1932-3, p. 74).

En efecto, su propósito es fortalecer al yo, hacerlo más independiente del superyó, ensanchar su campo de percepción y ampliar su organización de manera que pueda apropiarse de nuevos fragmentos del ello. Donde Ello era, Yo debe devenir (2007/1932-3, p. 74).

La cura, en este sentido, dependería de la fortaleza del yo para liberarse de los imperativos del superyó y poder aprehender contenidos inconscientes del ello, a los cuales de alguna manera hace propios. Este problema quedará abierto a revisión, pues la relación entre el ello y el superyó, como instancias entre las que media el yo, es sometida a diversas revisiones tanto por Freud como por Lacan.

Adicionalmente, en 1937, año tardío en la obra de Freud, el autor se ocupa del problema del final posible e imposible para un análisis. Allí considera dos posibles finales del mismo, concebidos a partir del nivel de cura que puede lograrse. Uno, si se quiere práctico, cuya condición radica en el atemperamiento del sufrimiento del sujeto y la superación de síntomas, angustias e inhibiciones; esta sería una vía, si se quiere terapéutica, por otro lado, abre una vía para pensar el final posible de un análisis en términos de saber, o un final investigativo, conclusivo de una elaboración de saber sobre la subjetividad y lo inconsciente. 
En la práctica es fácil decirlo. El análisis ha terminado cuando analista y paciente ya no se encuentran en la sesión de trabajo analítico. Y esto ocurrirá cuando estén aproximadamente cumplidas dos condiciones: la primera, que el paciente ya no padezca a causa de sus síntomas y haya superado sus angustias así como sus inhibiciones, y la segunda, que el analista juzgue haber hecho consciente en el enfermo tanto de lo reprimido, esclarecido tanto de lo incomprensible, eliminado tanto de la resistencia interior, que ya no quepa temer que se repitan los procesos patológicos en cuestión. (2007/1937, p. 222).

Lo anteriormente planteado nos permite ver la posibilidad de un final de análisis y la obtención de una cura tanto por la vía de lo terapéutico como por la vía de aquello propiamente analítico, pues cabe aclarar que si bien es cierto que la cura psicoanalítica implica una mejoría de los sufrimientos del sujeto, se instala más en el campo del saber y obedece a la singularidad, al deseo propio de aquel que se analiza, no hay, a diferencia de otras terapéuticas, una cura estándar, un saber válido para todos, ni un síntoma que tenga el mismo valor. Es decir que una cura psicoanalítica es una cura por la vía del uno por uno, de la elaboración de saber, es una cura de la ignorancia del yo del sujeto de aquello que lo constituye, su historia y los capítulos censurados de ella que Freud denominó represiones.

Sin embargo, en la cura psicoanalítica el levantamiento de los síntomas se topa con más de un obstáculo, por lo cual Freud forjó el concepto de resistencia, con ella aparece en el psicoanálisis la reacción terapéutica negativa como un impasse para la cura, mostrándonos el carácter inamovible de ciertos síntomas y la fijación del paciente a sus repeticiones, a la satisfacción pulsional que el síntoma puede proporcionar, así sea por la vía del sufrimiento. A propósito de esto afirma Freud:

Hay personas que se comportan de manera extrañísima en el trabajo analítico. Si uno les da esperanza y les muestra contento por la marcha del tratamiento, parecen insatisfechas y por regla general su estado empeora. Al comienzo, se lo atribuye a desafío, y al empeño por demostrar su superioridad sobre el médico. Pero después se llega a una concepción más profunda y justa. Uno termina por convencerse no sólo de que estas personas no soportan elogio ni 
reconocimiento alguno, sino que reaccionan de manera trastornada frente a los progresos de la cura. Toda solución parcial, cuya consecuencia debiera ser una mejoría o una suspensión temporal de los síntomas, como de hecho lo es en otras personas, les provoca un refuerzo momentáneo de su padecer; empeoran en el curso del tratamiento, en vez de mejorar. Presentan la llamada reacción terapéutica negativa. (2007/1923, p. 50).

Aparece, pues, un fenómeno en la clínica que muestra a Freud una dificultad consistente en que el proceso produce, en lugar del mejoramiento de los síntomas, su empeoramiento; estos casos complejizan más aun la formulación de una teoría de la cura en psicoanálisis, pues ponen en evidencia algo incurable, por lo menos desde el punto de vista de la voluntad consciente o inconsciente del paciente. En este sentido, "No hay duda de que algo se opone en ellas a la curación, cuya inminencia es temida como un peligro. Se dice que en estas personas no prevalece la voluntad de curación, sino la necesidad de estar enfermas" (Freud, 2007/1923, p. 50).

Con Lacan, a partir de su última enseñanza, aparece un problema que en el presente artículo solo será esbozado, es el problema del sinthome, como un nombre de lo incurable propio del síntoma desde la perspectiva que él imprime. Jacques Lacan no desconoce la dirección de la cura y las posibilidades terapéuticas del psicoanálisis como método terapéutico o investigativo, pero centra su atención en aquello que se comporta, a partir de una cierta inercia en la experiencia de análisis, una inercia que él ordena a partir de su concepción de lo real como imposible y de su teorización del goce propio del síntoma como irreductible. A propósito de esto, Jacques Alain Miller, en su curso Sutilezas analíticas afirma:

El sujeto en análisis está abonado al inconsciente, es decir que es susceptible de avances, de resistencias, y su estructura se presenta como estratificada. Está ese camino para hacer y ese camino dura, (...) pero, al mismo tiempo adoptar el punto de vista del sinthome es saber que hay, que habrá lo que no cambiará, es un límite inaugural aportado al furor sanandi, es lo incurable inscrito en la puerta de entrada: ¡No sueñes con curar, ¡No te jactes de tus éxitos terapéuticosi ¡Mira lo que no cambia! (Lacan, 2012, p. 95, énfasis en el original). 
Se abre pues la pregunta para lo incurable en el análisis, ya no por una resistencia del yo, sino por el encuentro con el síntoma en su naturaleza de goce, donde el principio del placer se revela como displacer y sufrimiento.

En la última enseñanza de Lacan, podemos extraer la siguiente pregunta de manera implícita: ¿cuál es el arreglo posible con lo incurable del síntoma? Es decir, una pregunta por la buena manera de usar el síntoma en cuanto que es incurable. En este punto solo es posible en el presente trabajo cerrar la cuestión introduciendo la cita de Lacan, enigmática aún, pero orientadora: "La buena manera es la que, habiendo reconocido la naturaleza de sinthome, no se priva de usarlo lógicamente, es decir, de usarlo hasta alcanzar su real, al cabo de lo cual el apaga su sed" (2008, p. 15).

\section{La resistencia hoy y fuera del dispositivo analítico}

El sujeto es un ser en permanente cambio y cado uno tiene sus propias especificidades y características de la época y el contexto en el que vive, esto exige al analista una formación que responda a las particularidades de cada época y los sujetos que ella produce. En este sentido, es necesario preguntarse: ¿qué ha cambiado en la resistencia desde la época de Freud hasta la época actual?, ¿hay nuevas formas de la resistencia hoy?, ¿cuáles serían esas nuevas formas y qué las causa?, ¿de qué manera se presentan y con qué fuerza?, ¿qué nuevas relaciones establecen con ella los analizantes y/o los analistas hoy?, ¿cuáles serían las nuevas formas de reconocerlas y abordarlas en el dispositivo analítico para ponerlas al servicio del proceso terapéutico? O, como diría Lacan: “¿Es la resistencia un fenómeno que sólo aparece en el análisis? ¿O bien es algo de lo que podemos hablar cuando el sujeto está fuera del análisis, incluso antes de llegar a él, o después de dejarlo? [o si] ¿Sigue teniendo sentido la resistencia fuera del análisis?" (1988a/1953-1954, p. 43). Dichas preguntas no son resueltas en el presente artículo, pero se plantean con el fin de provocar reflexiones adicionales al respecto. 


\section{Conclusiones}

A modo de conclusiones puede decirse, con Freud, que la resistencia se presenta a lo largo de todo el proceso analítico en la dirección de la cura, como una lucha entre dos fuerzas opuestas: una cuya meta es la salud y otra que la contraría. Ello implica una oposición entre unas fuerzas de repetición sintomática inconsciente y otras fuerzas que intentan recordar y saber sobre sí. De la resistencia puede decirse que es paradojal, pues al mismo tiempo es una posibilidad para la cura y también una amenaza para ella si no se le da un direccionamiento adecuado; ésta, además de ser un elemento paradójico, como quedó expuesto, también es un componente necesario para alcanzar la cura analítica, pues durante su auge y desarrollo es precisamente cuando se descubren los contenidos reprimidos de los cuales ella se alimenta, permitiendo, de esta manera, transformar lo reprimido inconsciente y la repetición sintomática en recuerdo consciente y bienestar subjetivo. Ha de tenerse en cuenta que es en el momento de la transferencia cuando más difícil resulta su vencimiento, pues la transferencia es la resistencia más difícil de vencer.

Con Lacan se dirá que la resistencia está del lado del analista cuando se deja orientar durante el análisis por sus prejuicios personales e interviene desde ellos. Éste teórico sitúa la resistencia en el momento de la transferencia, la cual cierra el inconsciente, pero al mismo tiempo lo abre, porque cuando ella se instala, se inicia el tiempo de la interpretación sobre los elementos que allí expone el analizante.

Debe decirse también, que la resistencia aparece durante el proceso de análisis, allí donde no puede decirse más, donde se agotan las palabras para nombrar el deseo, para capturarle en las letras y esto es estructural al aparato psíquico durante la práctica clínica, puesto que no todo puede ser dicho y algo quedará por fuera. Esa forma de la resistencia al final de análisis es estructural, en la medida en que hay una forma de ella que jamás podrá ser descifrada, puesto que hay algo de lo que no se podrá dar cuenta, lo Real, debido a que allí se agotan las palabras y aparece lo indecible. 
No todo se cura en una experiencia de análisis y esto tiene implicaciones para la formación del analista, pues está llamado a estar a la altura no solo de lo que puede curarse, sino también de lo incurable. Lo cual problematiza también el asunto de la cura desde otras disciplinas en una época cientificista que busca la eficacia terapéutica.

La cura en psicoanálisis es un tema complejo, que evolucionó tanto en Freud como en Lacan y aún no tiene una conceptualización definitiva en la medida en que todavía el psicoanálisis está en desarrollo. La cura se topa además con posibilidades y con limitaciones.

\section{Referencias}

Freud, S. (2005/1912). Sobre la dinámica de la trasferencia. En J. Strachey (Ed.), Obras Completas (J. L. Etcheverry, Trad., Vol. XII) (pp. 93-105). Buenos Aires, Argentina: Amorrortu.

Freud, Sigmund. (2005/1914). Recordar, repetir y reelaborar (nuevos consejos sobre la técnica del psicoanálisis, II). En J. Strachey (Ed.), Obras Completas (J. L. Etcheverry, Trad., Vol. XII) (pp. 145-157). Buenos Aires, Argentina: Amorrortu.

Freud, S.(2007a/1893-1895). Estudios sobre la histeria (Breuer y Freud). En J. Strachey (Obras Completas (J. L. Etcheverry, Trad., Vol. II) (pp. 1-25). Buenos Aires, Argentina: Amorrortu.

Freud, S. (2007b/1893-1895). Señorita Elisabeth Von R. En J. Strachey (Ed.), Obras Completas (J. L. Etcheverry, Trad., Vol. II) (pp. 151-194). Buenos Aires, Argentina: Amorrortu.

Freud, S. (2007/1903-1904). El método psicoanalítico de Freud. En J. Strachey (Ed.), Obras Completas (J. L. Etcheverry, Trad., Vol. VII) (pp. 235242). Buenos Aires, Argentina: Amorrortu.

Freud, S. (2007a/1916-1917). 19a Conferencia. Resistencia y represión. En J. Strachey (Ed.), Obras Completas (J. L. Etcheverry, Trad., vol. XVI) (pp. 262-276). Buenos Aires, Argentina: Amorrortu.

Freud, S. (2007b/1916-1917). 27 conferencia. La transferencia. En J. Strachey (Ed.), Obras Completas (J. L. Etcheverry, Trad., Vol. XVI) (pp. 392-407). Buenos Aires, Argentina: Amorrortu.

Freud, S. (2007c/1916-1917). 28 conferencia. La terapia analítica. En J. Strachey (Ed.), Obras Completas (J. L. Etcheverry, Trad., Vol. XVI) (pp. 408421). Buenos Aires, Argentina: Amorrortu. 
Freud, S. (2007/1920). Más allá del principio de placer. En J. Strachey (Ed.), Obras Completas (J. L. Etcheverry, Trad., Vol. XVIII) (pp. 1-62). Buenos Aires, Argentina: Amorrortu.

Freud, S. (2007/1923). El yo y el ello. En J. Strachey (Ed.), Obras Completas (J. L. Etcheverry, Trad., Vol. XIX) (pp. 1-66). Buenos Aires, Argentina: Amorrortu.

Freud, S. (2007/1932). 31 a conferencia. La descomposición de la personalidad psíquica. En J. Strachey (Ed.), Obras Completas (J. L. Etcheverry, Trad., Vol. XXII) (pp. 53-74). Buenos Aires, Argentina: Amorrortu.

Freud, S. (2007/1937). Análisis terminable e interminable. En J. Strachey (Ed.), Obras Completas (J. L. Etcheverry, Trad., Vol. XXIII) (pp. 211254). Buenos Aires, Argentina: Amorrortu.

Lacan, J. (1981/1953-1954). El seminario de Jacques Lacan, Libro 1: Los escritos técnicos de Freud. Barcelona, España: Editorial Paidós.

Lacan, J. (1988a/1953-1954). Capítulo II. Primeras intervenciones sobre el problema de la resistencia. En El seminario de Jacques Lacan, libro 1: Los escritos técnicos de Freud (pp. 37-51). Buenos Aires, Argentina: Paidós.

Lacan, J. (1988b/1953-1954). Capítulo III. La resistencia y las defensas. En El seminario de Jacques Lacan, Libro 1: Los escritos técnicos de Freud (pp. 53-65). Buenos Aires, Argentina: Paidós.

Lacan, J. (2002). La dirección de la cura y los principios de su poder. En Escritos 2 (pp. 559-615). Buenos Aires: Argentina: Siglo Veintiuno Editores.

Lacan, J. (2003/1964). Capítulo X, La presencia del analista. En El seminario de Jacques Lacan, Libro 11: Los cuatro conceptos fundamentales del psicoanálisis (pp. 129-141). Buenos Aires, Argentina: Paidós.

Lacan, J. (2008). Del uso lógico del sinthome, o Freud con Joyce. En El seminario de Jacques Lacan, libro 23, El sinthome (pp. 11-26). Buenos Aires, Argentina: Paidós.

Miller, J. A. (2012). V clínica del sinthome. En Sutilezas analíticas. Los cursos psicoanalíticos de Jacques-Alain Miller (pp. 83-96). Buenos Aires, Argentina: Paidós.

Velásquez, C. (2006). Lo que se resiste a una cura psicoanalítica. En Del deseo del analista (pp. 1-38). Medellín, Colombia: Editorial Universidad de Antioquia. 